\title{
Don't Bury the V/Q Scan: It's as Good as Multidetector CT Angiograms with a Lot Less Radiation Exposure
}

$\mathbf{W}_{\text {it }}$ o angiographic studies for pulmonary embolism (PE) diagnosis. This has been embraced by clinicians and most diagnostic radiologists who are more comfortable with an anatomic demonstration of whether a clot is present or not as compared with looking at ventilation-perfusion (V/Q) mismatches on a lung scan (1). As a result, the number of multidetector $\mathrm{CT}$ angiograms (MDCTA or CTA) performed in the past few years has greatly increased with little regard for radiation dose, particularly to the breasts of young women. In addition, sufficient evidence has now been accumulated to confirm that V/Q scintigraphy is as accurate as CTA, making it a viable alternative when studying patients for PE.

In a recent Invited Perspective in The Journal of Nuclear Medicine, Dr. Arnold Strashun comments on the MDCTA versus V/Q issue and concludes that "were it not for definite allergic and nephrotoxic risks of contrast media and the added radiation burden of MDCTA, the ventilation/perfusion scan would virtually disappear from the diagnostic algorithm for pulmonary embolism" (2). The CTA problems mentioned by Strashun are innate to the modality and cannot be mitigated. Therefore, it appears quite clear that V/Q imaging

Received Oct. 17, 2007; revision accepted Nov. 19, 2007.

For correspondence or reprints contact: Leonard M. Freeman, MD, Montefiore Medical Center, 111 East 210th St., Bronx, NY 10467.

E-mail: Ifreeman@montefiore.org

COPYRIGHT @ 2008 by the Society of Nuclear Medicine, Inc.

DOI: 10.2967/jnumed.107.048066 will continue to be a very important part of the PE diagnostic algorithm for the foreseeable future. In addition to substantial data confirming that V/Q sensitivity is comparable to that of MDCTA, the radiation risk aspect (particularly to the female breast) requires much stronger emphasis. Young women represent a very significant segment of the population being studied. As pointed out in the recent American College of Radiology (ACR) white paper (3), dose calculation is very complex because absorption in each organ is very variable from patient to patient. Breast radiation estimates made using 4-slice CT vary from 20 to $60 \mathrm{mSv}(4-6)$, whereas that from V/Q is approximately $0.28-0.9$ $\mathrm{mSv}$ (7). A recent report by Einstein et al. (8) estimated that 64-slice chest CTA delivers a dose of $50-80 \mathrm{mSv}$ to the breast. These reports indicate an enormous 65- to 250-fold difference between the 2 procedures. Average estimates generally quoted are a 70 - to 100 -fold difference. Comparatively, a 2-view mammogram is associated with $3 \mathrm{mSv}$ (4), which makes the CTA radiation dose approximately 10 to 20 times greater. In addition, the estimated radiation exposure from CTA suggests that a nonnegligible increase in lifetime attributable risk of cancer exists, particularly to the breasts of young women ( 1 in 143 for a 20-y-old woman and 1 in 284 in a 40y-old woman) (8). The International Commission on Radiation Protection (ICRP) has reported that CT doses can exceed limits shown to result in an increase in cancer risk (9). These are facts about which we all must be very concerned and be professionally obligated to deal with them. To address this great concern, the ACR white paper (3) strongly emphasizes that it is the responsibility of the imaging physician to be fully educated concerning the radiation risks associated with each procedure and, in turn, educate the clinician requesting the procedure. Presenting them with diagnostically equivalent options also is part of this educational process. This is certainly of paramount importance and pertinence in the MDCTAvs. V/Q issue.

One advantage of CTA over V/Q mentioned by Strashun is that a significant number of patients have alternate anatomic diagnoses made that may be the cause of the patient's symptomatology (2). Most of these serendipitous findings, such as small lung nodules, generally are not related to the patient's acute problem. However, occasional significant findings, such as dissecting aneurysms or pneumothorax, may be detected (10).

In addition to the most important radiation issue, there are several factors that should be considered when deciding between a CTA and V/Q study. These are (a) Is one of the studies clearly superior to the other in the overall context of diagnosing PE as well as in combination with objective clinical assessment (pretest probability)? (b) Are both studies equally available on a 24-h/7-d schedule? (c) Do the physicians interpreting the study possess the appropriate expertise?

\section{DIAGNOSTIC ACCURACY OF MDCTA VS. V/Q IMAGING AND ITS USE IN COMBINATION WITH OBJECTIVE CLINICAL ASSESSMENT}

Interestingly, the long-awaited results of the PIOPED II study published 
on June 1, 2006, in the New England Journal of Medicine (11) do not clearly support the superiority of CT angiography over V/Q scanning for the diagnosis of PE.

The definition of PE present in PIOPED II was based on composite reference standards that included a positive (high probability), negative, or low-probability V/Q scan, pretest probability as based on a positive $(>6)$, intermediate $(2-6)$, or negative $(<2)$ Wells score (12), and the results of venous ultrasonography for deep venous thrombosis. PE was also diagnosed on the basis of results of digital subtraction angiography. The V/Q study (along with the other reference criteria) represented a significant component of what constituted "truth" (final decision as to whether or not the patient actually had PE).

In PIOPED II, 824 patients with a positive or negative reference standard were studied with CTA. The overall sensitivity of CTA for the diagnosis of PE was $83 \%$ and the specificity was $96 \%$. The overall positive predictive value (PPV) was $86 \%$, and the negative predictive value (NPV) was $95 \%$. These values are comparable to V/Q statistics, where high-probability scans are associated with a $>85 \%$ likelihood of $\mathrm{PE}$ and low-probability results equate to a $<20 \%$ likelihood of PE (13).

One of the most important pieces of information derived from both the original PIOPED I as well as the PIOPED II studies is the critical importance of objective clinical assessment to the final diagnosis. To create objective consistency in assessing and reporting pretest probability, we have encouraged all of our clinicians - particularly those in the emergency department (ED) - to use the Wells score (12). Both CTA and V/Q imaging lose considerable value when there is discordance between objective clinical assessment and test results. In PIOPED II, the PPV of a positive MDCTA was only $58 \%$ when clinical probability was low (11). Very similar results in PIOPED I for the V/Q scan showed a PPV of 56\% when such discordance was present (13). In fact, the final sentence in PIOPED II states "additional testing is necessary when clinical probability is inconsistent with the imaging results" $(11)$.

After the publication of the PIOPED II results, the involved investigators published similar editorials in the American Journal of Medicine and Radiology proposing diagnostic pathways to study PE based on objective clinical assessment (14). The d-dimer rapid enzyme-linked immunosorbent assay was used as the primary study to help determine what subsequent pathway to follow. A low-probability objective clinical assessment followed by a negative d-dimer effectively rules out PE in most instances, and no diagnostic imaging study may be needed.

Some of the PIOPED II investigators recently performed a retrospective analysis of their data and have validated criteria for the PIOPED II "very low probability" (<10\% PPV) (15). These criteria were described by Gottschak 5 y earlier (16), reviewed by Freeman et al. (17), and were validated by the current review, which shows that "very low probability" lung scans (defined as $<10 \%$ PPV) in combination with low- probability objective clinical assessment reliably excludes PE (15). Scintigraphic very low probability criteria include such things as nonsegmental perfusion abnormalities, stripe sign, solitary triple matched defect in the middle or upper lung zones, and so forth (15).

The approach that we have used at Montefiore certainly recognizes objective clinical assessment as a very key factor but does not use it to determine which imaging study should be performed. Instead, we use the plain chest radiograph as the main decision-maker in most patients. If abnormal findings, such as opacities, atalectasis, or pleural effusions exist, CTA is recommended. If the radiograph is negative (which it most often is), V/Q scintigraphy is the likely examination to follow, as it can be reliably interpreted. This has worked out quite well. One of our ED physicians related to me that clinicians feel "more comfortable" with a negative or normal CTA rather than a low-probability V/Q scan. When I pointed out the $83 \%$ sensitivity results from PIOPED II and its close similarity to V/Q data, it was a revelation. The $17 \%$ false- negative CTAs were believed, likely to represent very peripheral, insignificant emboli that don't require any treatment (11). This is a controversial point, as some investigators believe that these peripheral PEs should be detected and treated (18).

The language used for interpretation is important and it would probably be more accurate and understandable if the term "no evidence of PE" was used instead of a normal CTA or very low probability V/Q scan.

One final point to consider in support of V/Q imaging is the prognostic value of a low-probability interpretation. In 787 patients in 3 different published series (19-21), the incidence of serious subsequent related events over a 6-mo or longer follow-up was $<1 \%$. This is an additional factor that should be very comforting to the referring physician.

\section{RELATIVE AVAILABILITY OF MDCTA AND V/Q SCINTIGRAPHY}

There is a need to rapidly triage patients in a busy emergency room. A visit to any large ED makes this need quite evident. A patient may present with some dyspnea, chest pain, or other finding where PE may be a consideration, albeit a minor one. Despite a low Wells score, the ED physician would welcome a negative confirmatory imaging study before discharging a patient and potentially putting the patient into an extremely unlikely, but ever-existent, life-threatening event. A rapidly obtained CTA often is the easier choice.

Lack of availability of V/Q imaging during evening hours and weekends is a major factor and, unfortunately, is most often the determinant of the choice between which procedure is chosen. Almost all medical centers and community hospitals offer aroundthe-clock CT services. Often, the CT scanner is located in close proximity to the $\mathrm{ED}$, which allows the clinician 
to obtain studies fairly rapidly. $\mathrm{Nu}-$ clear medicine services are available on evenings and weekends in relatively few institutions as the volume of work does not justify an on-site presence of a technologist. In institutions in which nuclear medicine services are available on a 24-h/7-d basis, the technologist must be called in and must prepare the radiopharmaceuticals before the study. At Montefiore, we have done this effectively for many years as it takes an hour or less from the time of the initial request until the technologist arrives on site. In institutions in which scintigraphy is not available at night, the possibility of temporarily treating a patient with a single dose of low-molecular-weight heparin can be considered if there is a need to avoid MDCTA; such as in young women. This dose is, apparently, good for 6-8 h and can tide a patient over until a V/Q study is performed in the morning.

\section{EXPERTISE OF RADIOLOGY AND NUCLEAR MEDICINE RESIDENTS IN INTERPRETING V/Q STUDIES}

Radiologists, as a whole, do not feel as comfortable interpreting radionuclide images as compared with other areas of diagnostic imaging. I believe that this is an important factor as to why most chest radiologists and body imagers, in general, encourage the use of CTA instead of the V/Q scan. Fortunately, our diagnostic radiologists at Montefiore share my viewpoint and have successfully reversed the trend in the unacceptably high number of MDCTAs performed. In early January 2007, our Chief of Diagnostic Radiology, Chief of Chest Radiology, and I met with our ED Director and several staff physicians to discuss the problem. A surgical grand rounds was also devoted to this subject. My fellow radiologists also have been very concerned about the enormous rise in CTAs being performed; particularly those in patients with low pretest probabilities (Wells score of $\leq 2$ ). Many of these have been performed hoping to have a negative study to comfortably allow discharge. It was quite evident that they had little or no knowledge of the radiation dose problem. Additionally, when presented with the PIOPED II data concerning the $83 \%$ sensitivity of MDCTA, they were more willing to cut back in favor of V/Q imaging. As indicated earlier, in addition to the Wells score, the chest $\mathrm{X}$-ray is now used as the initial examination. If negative, a V/Q scan is requested, and if positive showing opacities or fluid, a CTA is performed. This has worked very well with a reversal of the previous years' trend. The numbers for the first 8 mo of 2007 were quite revealing when contrasted with any 8-mo period in the prior $2 \mathrm{y}$. Specifically, when compared with the last 8 mo of 2006, CTA studies for PE dropped from 963 to 702, whereas V/Q studies rose from 438 to 779 . This decrease of $27.1 \%$ in CTAs associated with an increase of $82 \%$ in V/Q studies could be used as a model of what can be achieved with a lot of communication between radiologists, nuclear medicine physicians, and clinicians.

\section{SIGNIFICANT ROLE OF V/Q EVEN WHEN MDCTA IS THE PRIMARY PROCEDURE USED}

Being well aware of my great interest in this area, several of my colleagues have advised me that I was probably right and they agree with me, but that I am "fighting a losing battle." I would like to believe that, as a patient advocate for all of the above reasons, this is not true. Even in those institutions that persist in using MDCTA as their sole study for PE, contrast allergy and nephrotoxicity problems as well as claustrophobia and obesity are areas where $V / Q$ imaging cannot be readily avoided. Radionuclide imaging has also been the primary examination used in pregnant patients. A low-dose, perfusion-only study using $37 \mathrm{MBq}$ (1 $\mathrm{mCi}$ ) of ${ }^{99 \mathrm{~m}} \mathrm{Tc}$-labeled macroaggregated albumin has proven to be a very successful screening procedure in this patient population.

In addition, there are 2 areas in which follow-up V/Q is of importance, and the use of repeat CT is certainly unwarranted:
- It is most beneficial to follow all positive CT studies with a baseline V/Q study for the purpose of long-term follow-up. Patients may present at a future time with recurrent suspicion of a new $\mathrm{PE}$. Although CT findings have been described to distinguish old from new clots, this is not always easy. Serial V/Q studies showing resolution or the lack thereof significantly facilitate such important decisions (22-24).

- A baseline V/Q study should be routinely performed in patients with deep venous thrombosis (DVT). The incidence of silent $\mathrm{PE}$ in these patients is estimated to be $38 \%$ or greater. $(25,26)$. In a patient who has been anticoagulated and, subsequently, presents with a suspicion of $\mathrm{PE}$, neither a positive MDCTA or V/Q study will be able to judge whether the embolus has occurred before or after the start of the anticoagulation. If the latter, disruption of the inferior vena cava with an "umbrella" procedure may be warranted. If the PE was present at the time of DVT diagnosis, continuation of anticoagulant therapy will suffice.

\section{CONCLUSION}

The enormous increase in the number of MDCTAs for studying possible pulmonary embolic disease is a trend that must be reversed. This is necessary not only because of unacceptably high radiation exposure-particularly to the breasts of young women-but also because of the erroneous belief that it is a superior diagnostic tool as compared with V/Q imaging. In many instances, the considerably lowerradiation-exposure dose associated with the radionuclide study makes it a preferable choice. It is the responsibility of the imaging physician to be knowledgeable about the relative value and the benefit-to-risk ratio of each procedure to properly advise the referring physician. Paramount to this knowledge base and discussion is an in- 
timate knowledge of the relative radiation exposure associated with each procedure. As recommended by the American College of Radiology's white paper on radiation dose in medicine, this must be emphasized in all training programs.

Most medical centers do not offer "after hours" nuclear medicine services on nights and weekends. This fact as well as the 24-h ready availability of CT scanners and the erroneous belief that it is a more sensitive study has played a role in influencing decisions in favor of MDCTA to study possible embolic disease. Although this trend (greatly influenced by economic factors) may be difficult to reverse, it is important that we become patient advocates and try. It is my belief that a plain chest radiograph can be used to determine which of these 2 procedures should be performed with a normal or near-normal radiograph favoring V/Q scintigraphy, whereas a positive radiograph should be followed by MDCTA.

It is important for imaging physicians and clinicians alike to recognize that logistical, economic, and personal bias factors are influencing the decision being made rather than any convincing evidence that MDCTA is superior to V/Q imaging in this diagnostic area. It also is the inherent responsibility of all radiologists and nuclear medicine physicians to educate our referring clinicians about the excessive radiation exposure associated with MDCTA, particularly to the female breast.
Leonard M. Freeman

Montefiore Medical Center Albert Einstein College of Medicine Bronx, New York

\section{REFERENCES}

1. Goldhaber SZ. How chest CT for the diagnosis of pulmonary embolisn (PE) has changed my professional life: reflections from a PE doctor. Semin Roentgenol. 2005;40:8-10.

2. Strashun AM. A reduced role of V/Q scintigraphy in the diagnosis of acute pulmonary embolism. J Nucl Med. 2007;48:1405-1407.

3. Amis ES, Butler PF, Applegate KE, et al. American College of Radiology white paper on radiation dose in medicine. $J$ Am Coll Radiol. 2007;4:272-284.

4. Parker MS, Hui FK, Camacho MA. Female breast radiation exposure during CT pulmonary angiography. Am J Radiol. 2005;185:1228-1233.

5. Cook JV, Kyriou J. Radiation from CT and perfusion scanning in pregnancy [letter]. Br Med J. 2005;331:350.

6. Task Group on Control of Radiation Dose in Computed Tomography: a report of the International Commission on Radiological Protection. Ann ICRP. 2000;30:7-45.

7. Radiation dose to patients from radiopharmaceuticals. Ann ICRP. 1998;28:1-126.

8. Einstein AJ, Henzlova MJ, Rajagopolan S. Estimating risk of cancer associated with radiation exposure from 64-slice computed tomography coronary angiography. JAMA. 2007;298:317-323.

9. The International Commission on Radiological Protection. Managing patient dose in computed tomography: a report of the International Commission on Radiological Protection-ICRP publication 87. Ann ICRP. 2000;30:7-45.

10. Schoepf U, Costello P. CT angiography for diagnosis of pulmonary embolism: state of the art. Radiology. 2004;230:329-337.

11. Stein PD, Fowler SE, Goodman LR, et al. Multidetector computed tomography for acute pulmonary embolism. N Engl J Med. 2006;354:2317-2327.

12. Wells PS, Anderson DR, Rodger $M$, et al. Excluding pulmonary embolism at the bedside without diagnostic imaging: management of patients with suspected pulmonary embolism presenting to the emergency department by using a simple clinical model and D-dimer. Ann Intern Med. 2001;135:98-107.

13. Value of the ventilation/perfusion scan in acute pulmonary embolism: results of the prospective investigation of pulmonary embolism diagnosis
(PIOPED): the PIOPED Investigators. JAMA. 1990;263:2753-2759.

14. Stein PD, Woodard PK, Weg JG, et al. Diagnostic pathways in acute pulmonary embolism: recommendation of the PIOPED II investigators. Radiology. 2007;242:15-21.

15. Gottschalk A, Stein PD, Sostman HD, et al. Very low probability interpretation of V/Q lung scans in combination with low probability objective clinical assessment reliably excludes pulmonary embolism: data from PIOPED II. J Nucl Med. 2007;48: 1411-1415.

16. Gottschalk A, Stein PD, Goodman LR, Sostman HD. Overview of prospective investigation of pulmonary embolism diagnosis II. Semin Nucl Med. 2002;32:173-182.

17. Freeman LM, Krynycki B, Zuckier LS. Enhanced lung scan diagnosis of pulmonary embolism with the use of ancillary scintigraphic findings and clinical correlation. Semin Nucl Med. 2001;31: 143-157.

18. Tunariu N, Gibbs JR, Win Z. Ventilation-perfusion scintigraphy is more sensitive than multidetector CTPA in detecting chronic thromboembolic pulmonary disease as a treatable cause of pulmonary hypertension. J Nucl Med. 2007;48:680-684.

19. Smith R, Maher JM, Miller RI, et al. Clinical outcomes of patients with suspected pulmonary embolism and low probability aerosol-perfusion scintigrams. Radiology. 1987;164:731-733.

20. Kahn D, Bushnell DL, Dean R, et al. Clinical outcome of patients with a "low probability" of pulmonary embolism on ventilation-perfusion lung scan. Arch Intern Med. 1989;149:377-379.

21. Rajendran JG, Jacobson AF. Review of 6-month mortality following low probability lung scans. Arch Intern Med. 1999;159:349-352.

22. Alderson PO, Dzebolo NN, Biello DR, et al. Serial lung scintigraphy: utility in diagnosis of pulmonary embolism. Radiology. 1973;149:797-802.

23. Wartski M, Collignon MA. Incomplete recovery of lung perfusion after 3 months in patients with acute pulmonary embolism treated with antithrombic agents: THESEE Study Group. $J$ Nucl Med. 2000;41:1043-1048.

24. Gottschalk A. The chronic perfusion defect: our knowledge is still hazy, but the message is clear. J Nucl Med. 2000;41:1049-1050.

25. Moser KM, Fedullo PF. LitteJohn JK, Crawford R. Frequent asymptomatic pulmonary embolism in patients with deep venous thrombosis. JAMA. 1994;271:223-225.

26. Monreal M, Ruiz J, Olazabal A, et al. Deep venous thrombosis and the risk of pulmonary embolism. Chest. 1992;102:677-681. 